\title{
$\operatorname{arCOS} D E S I G N$
}

\section{A importância da estatística para a pesquisa em Design: algumas recomendações}

Raimundo Lopes Diniz, D. Sc. (UFMA, Brasil)

diniz@ufma.br

Campus Universitário do Bacanga - CCET

Bloco 06, sala 216, São Luís, MA, CEP: 65080-580

Lívia Flávia de Albuquerque Campos, Ma. (UNESP, Brasil)

liviaflavia@gmail.com

Patrícia Régia Sodré Nicácio (UFMA, Brasil)

patriciaregia@gmail.com

Osmar Lopes da Silva Filho (NEEP/UFMA, Brasil)

olfilho@hotmail.com 


\section{A importância da estatística para a pesquisa em Design: algumas recomendações}

Resumo: O presente artigo apresenta uma discussão a respeito da importância da estatística ao processo de pesquisa em design, além de recomendações quanto ao planejamento, incluindo o uso e aplicação, de ferramentas da estatística. Pode-se considerar, ainda, que em decorrência da pesquisa em design ter uma abrangência multifacetada, a estatística não pode ser encarada como uma obrigação de uso em termos de produção do conhecimento.

Palavras-chave: pesquisa em design, análise de dados, processos estatísticos

\section{The importance of statistics for research in Design: recommendations for actions.}

Abstract: This article presents a discussion related to the importance of statistics to the process of design research, and recommendations regarding the statistics design, including the use and application of statistical tools. It is worth mentioning that the design research has a scope multifaceted and, so the statistic processes can't be considered as principal criteria to use in terms of knowledge production.

Key words: design research, data analysis, statistical processes 


\section{Introdução}

Design pode ser definido como "o processo de adaptação do ambiente artificial às necessidades físicas e psíquicas dos homens na sociedade" (LОвасн, 2001, p. 14). Ou ainda como apresenta Coelho (2008, p. 187), o Design:

[...] é essencialmente uma práxis, que acompanhada de teorias (para fundamentação e crítica), tem como tarefa dar forma a artefatos, considerando um projeto previamente elaborado com uma finalidade objetiva e específica. É um campo amplo de atividades (desempenhos) especializadas, de caráter técnico, científico e artístico, que se ocupam em organizar, classificar, planejar, conceber, projetar, configurar, sistemas de informação, objetos bidimensionais (gráficos), tridimensionais (volumétricos), virtuais, ambientes ou espaços para produção industrial e/ou artesanal.

Ressalta-se, ainda, que essas atividades (CoelHo, 2008, p. 187):

[...] levam em consideração no desenvolvimento projetual não apenas a produtividade do processo de fabricação, mas também as questões de uso, função (objetiva e subjetiva), produção, mercado, utilidade, qualidade formal e estética (fruição do uso), buscando equacionar sistêmica e funcionalmente, fatores sociais, culturais, antropológicos, ecológicos, ergonômicos, tecnológicos e econômicos. O processo de configuração, portanto, leva em consideração critérios estéticos e extra-estéticos no estabelecimento de parâmetros - critérios pelos quais ele será considerado adequado ou não para uma finalidade específica de trabalho -, sem perder de vista a relação com o ser humano no aspecto de uso ou de percepção, buscando atender as necessidades e contribuir para o bem estar e conforto individual e/ou coletivo.

Segundo Bonsiepe (2012, p. 19) "o Design, o contrário de outras disciplinas universitárias, não se orienta, prioritariamente para a geração de novos conhecimentos científicos, mas visa às práticas da vida cotidiana”. Entretanto, “apesar da abordagem diferente entre ciência e design, já que o design é caracterizado por um olhar para o mundo da perspectiva projetual, e a ciência o encara pela perspectiva do reconhecimento, é possível gerar conhecimentos e realizar pesquisas na área de design”. Para o autor, avanços nesse sentido são 
importantes e acrescenta que na atual fase histórica caracterizada "por uma intensa inovação científica, tecnológica e industrial, torna-se mais evidente a necessidade de gerar conhecimentos a partir da perspectiva do projetar, sobretudo tratando-se de problemas complexos que excedem o know-how de uma disciplina particular" (вONSIEPE, 2012, p. 19).

Van der Linden (2010) sugere que a pesquisa em design, principalmente no Brasil, é relativamente jovem e, ainda, que esta encontra-se em fase de consolidação, com pouco arcabouço teórico, em contínua discussão. O autor evidencia que a dificuldade de tal consolidação pode ser encontrada considerando-se a dimensão multifacetada da pesquisa em Design e a sua inserção institucional em diferentes áreas. Mesmo assim, a pesquisa em design vem produzindo conhecimento ao longo do tempo.

A respeito do conhecimento, Lakatos e Marconi (1992, p. 17) definem que "o conhecimento científico é real (factual), porque lida com a ocorrência dos fatos. Constitui um conhecimento contingente, pois suas proposições e hipóteses têm sua veracidade ou falsidade conhecida através da experimentação e não apenas pela razão, como no conhecimento filosófico." O conhecimento científico é ainda:

sistemático, já que se trata de um saber ordenado logicamente, formando um sistema de ideias (teoria) e não conhecimentos dispersos e desconexos. Possui a característica da verificabilidade, a tal ponto que as afirmações (hipóteses) que não podem ser comprovadas não pertencem ao âmbito da ciência. Constituem-se em um conhecimento falível em virtude de não ser definitivo, absoluto ou final, por este motivo, é aproximadamente exato: novas proposições e o desenvolvimento de técnicas podem reformular o acervo de teoria existente (LAKATOS; MARCONI, 1992, p. 17).

Lakatos e Marconi (1992) afirmam, ainda, que os processos estatísticos apresentam como objetivo principal a obtenção, a partir de conjuntos complexos de dados, de representações simples que podem servir de referencial para verificações simplificadas da existência de determinados problemas (fenômenos) e suas relações. Neste âmbito, a pesquisa em design, independente de sua faceta, pode utilizar a estatística como aliada na geração de conhecimento científico, no sentido de coleta e validação de dados.

Desta maneira, o presente artigo pretende abordar a importância da estatística para a pesquisa em design, apontando recomendações básicas quanto às estratégias de uso e aplicação. 


\section{Pesquisa em design}

Mesmo afirmando que a pesquisa ou investigação científica apresenta inúmeros conceitos, nos diferentes campos do conhecimento humano, Marconi e Lakatos (1996) apontam um consenso. Para os autores, a pesquisa é de suma importância no campo das ciências sociais, com o intuito de fornecer soluções para problemas coletivos. Ressalta-se o fato de que no Brasil, em termos de pesquisa e pós-graduação, a área Design ou Desenho Industrial encontra-se na grande área "Ciências Sociais Aplicadas", conforme a Coordenação de Aperfeiçoamento de Pessoal e Nível Superior (CAPEs). A pesquisa social é processual e lança mão de metodologia científica como um meio de investigação da realidade social (MARCONI; LAKATOS, 1996).

A base ou ponto de partida para a pesquisa e o seu sucesso é sempre uma teoria, sendo esta utilizada como instrumento da ciência, fundamentando os tipos de dados encontrados, servindo de sustento para sua análise (MARCONI; LAKATOS, 1996). A revisão da literatura ou a fundamentação teórica, portanto, valida os resultados encontrados, gerando assim a descoberta de princípios básicos e conhecimento aplicável, independentemente do tipo de pesquisa.

Conforme Moraes e Mont'Alvão (2010), de maneira geral, há dois tipos de pesquisa: 1. Pesquisa descritiva, a qual requer o reconhecimento e interpretação da realidade, sem interferência ou modificação, isto é, o objetivo principal é a descrição de fenômenos indagando o seu acontecimento, incluindo a sua relação com eventuais variáveis; 2 . Pesquisa experimental, a qual realiza a manipulação deliberada de algum aspecto da realidade, sob um planejamento pré-definido, a fim de observar de que modo ou em decorrência de quê os fenômenos acontecem, assim com sua possível correlação com determinadas variáveis, partindo de hipóteses que serão confirmadas ou refutadas. Portanto, o tipo de pesquisa será definido de acordo com o reconhecimento e formulação do(s) problema(s).

Parafraseando Marconi e Lakatos (1996, p. 16) "a pesquisa sempre parte de um tipo de problema, de uma interrogação (...)”. Formular um problema significa reduzir a situação problemática aos seus aspectos mais significativos e solucionáveis, desta forma, delineando-o (MORAES; MONT'ALVÃo, 2010). Para Löbach (2001), o trabalho do designer é encontrar uma solução para o problema, ou seja, o processo de design é tanto um processo criativo (talvez, a principal característica do profissional Designer) como um processo de solução de problemas, incorporando as características ou requisitos que eventualmente possam satisfazer a(s) necessidade(s) dos usuários. Bonsiepe (2012) aponta que é completamente possível a geração de conhecimentos por meio de pesquisas na área de design. Por outro lado, Bomfim (1998), descreve que 
Design é em sua essência uma práxis, a qual é normalmente acompanhada de teorias (de fundamentação e de crítica), sendo a teoria e a práxis partes de um mesmo processo que objetiva a solução ideal para um problema.

Para Niemeyer (2008), a pesquisa em design só é possível a partir da identificação de um problema, em seguida, serão considerados determinados procedimentos na busca da compreensão do mesmo e, consequentemente, a sua solução. Esse processo é norteado por um planejamento adequado, no qual serão construídas e aplicadas as possibilidades de operação dos dados, sendo os resultados encontrados submetidos a critérios de verificação e, só então, podem vir a integrar o arcabouço teórico da área. A autora afirma, também, que tais teorias tem a possibilidade de serem aplicadas em tecnologias próprias para a solução da "situação problema" original, sendo esta temporária, pois novos impasses ou desafios poderão trazer novas críticas e revisões, resultando em novos avanços teóricos.

A ciência em design, assim como em outras áreas, aplica a metodologia científica para lidar com problemas próprios à área e, assim, formam a base para o seu avanço e consolidação (NIEMEYER, 2008), para a aplicação dos achados ao aperfeiçoamento da atuação projetual e o amplo alcance dos objetivos de realização, seja nos âmbitos econômicos, quanto nos ambientais, culturais, éticos etc. Busca-se a consistência dos fundamentos e a eficácia das soluções de projeto propostas.

Na prática o que se pode constatar é que o cenário atual quanto à produção científica nacional em Design está em expansão. O Congresso Brasileiro de Pesquisa e Desenvolvimento em Design - P\&D Design ocorre desde 1994 e representa o potencial científico do design brasileiro; publicou, até $2008 \mathrm{em}$ seus anais, mais de 1.900 artigos (PASCHOARELLI et al, 2010). Essa produção tem sido expressiva e na sua última edição, ocorrida em 2012, foram submetidos 947 artigos, dentre estes, 876 artigos foram aprovados e 625 foram apresentados como comunicação oral. O que demonstra a qualidade técnica e científica de grande parte dos papers aprovados. Dos restantes, 251 foram apresentados como posters. Do total de trabalhos, 568 foram artigos completos e 57 foram submetidos como artigos de iniciação científica.

O cenário internacional da publicação em Design também está em expansão e tem apresentado exigência refinados métodos de análise de dados e análises estatísticas tem sido contempladas em boa parte dos estudos apresentados. Uma das principais revistas internacionais de Design a International Journal of Design, com fator de impacto de 1.253, apresentou, no ano de 2011, $31 \%$ de suas publicações com resultados que contemplaram avaliações estatísticas. Em uma de suas edições de 2013, 41\% das publicações apresentaram análises estatísticas importantes, incluindo estudos complexos com Análise 
de variância (KIM; CHIO, 2013), Análise fatorial (FORSLUND, et. al, 2013) e Correlações (BLIJLEvens et al., 2013).

Observa-se neste contexto, que diante do atual cenário de expansão da pesquisa científica em Design, a compreensão dos processos estatísticos e sua correta aplicação podem contribuir como norteadores da validação de resultados encontrados, fundamentando eventuais soluções aos problemas delineados.

\section{Os processos estatísticos e sua relevância para a pesquisa em design}

Os processos estatísticos permitem reduzir sistematicamente fenômenos a termos quantitativos e a manipulação estatística permite comprovar as relações dos fenômenos entre si, e obter generalizações sobre a natureza, ocorrência e significado, permitindo a verificabilidade. $O$ papel da estatística para a pesquisa é, antes de tudo, fornecer uma descrição quantitativa e deve ser considerado mais do que um meio de descrição racional; mas também, um método de experimentação e de prova, um método de análise (LAKATOS; MARCONI, 1992).

Beiguelman (2002, p. 3) ressalta a importância da estatística na pesquisa. Segundo o autor, é impraticável o estudo com toda uma população, portanto, as conclusões a respeito das características populacionais que são capazes de serem representadas por valores numéricos são obtidas por intermédio da análise de amostras da população em estudo. Denomina-se Inferência Estatística a "parte da estatística que se dedica à criação e emprego de métodos para obter conclusões a respeito de uma população por meio de uma amostra dela retirada". Complementa, ainda, que "no estudo destas amostras não se obtém os verdadeiros valores das características da população e sim uma estimativa dos mesmos". A importância disso refere-se ao fato de que "tais estimativas raramente são idênticas aos valores da população (geralmente desconhecidos), mas será possível por meio do estudo de uma amostra, afirmar, com grande probabilidade de acerto que o valor da característica da população está contido dentro de um determinado intervalo".

Em inferência estatística, preocupa-se em extrair conclusões sobre um grande grupo de objetos ou eventos que ainda estão por ocorrer, com base na observação de poucos objetos ou fatos que tenham ocorrido. A estatística fornece ferramentas que formalizam e padronizam os procedimentos para obter tais conclusões. Os procedimentos de inferência estatística introduzem ordem em qualquer tentativa de extrair conclusões da evidência proporcionada por amostras (SIEgEL; CASTELLAN, 2006). 
Para a pesquisa em design, como para outras áreas de estudo, a importância dos testes estatísticos está em, como afirmam Siegel e Castellan (2006), se a partir da evidência que se coletou, pode-se ter confiança no que se concluiu sobre o grande grupo, no qual somente poucos objetos foram observados (SIEGEL; CASTELlan, 2006). Na prática de pesquisa em Design, a inferência estatística permite, a partir de um conjunto de dados, concluir a respeito de uma determinada população, por exemplo, mediante a pesquisa com uma amostra de usuários de um produto/sistema, concluir a respeito de toda a população de usuários deste produto/sistema.

Tendo em mente que, para realizar a inferência estatística é necessário realizar a descrição sucinta dos dados amostrais, para isso, a "Estatística descritiva dedica-se às técnicas empregadas para descrever o comportamento médio e a variabilidade das informações contidas nas amostras" (BEIGUELMAN, 2002, p.3).

Tendo em mente que, para realizar a inferência estatística, é necessário, após a coleta, realizar a descrição sucinta dos dados amostrais, para isso é importante conhecer a Estatística Descritiva, a qual, segundo Beiguelman (2002, p.3), "dedica-se às técnicas empregadas para descrever o comportamento médio e a variabilidade das informações contidas nas amostras”. Estatísticas descritivas, como o nome indica, descrevem os dados sem dizer nada sobre a população maior. Já a Estatística inferencial permite se tirar algumas conclusões ou inferir sobre uma população maior acima e além de sua amostra (TULLIS; ALBERT, 2008).

Parte-se da Distribuição de frequências para variáveis contínuas (ver Beiguelman (2002, p. 4-9) para saber mais sobre variáveis continuas e discretas). Distribuição de frequências é a organização dos dados em classes de um mesmo intervalo. Uma distribuição de frequências pode ser mais facilmente entendida quando representada graficamente, e para isso vale-se do Histograma ou do Polígono de frequências (beiguelman, 2002).

A figura 1 apresenta o Polígono de frequências de dados de força manual em Newtons (N) (Contração Voluntária Máxima), de 90 sujeitos do gênero masculino, em movimento no sentido da posição pronada para a posição neutra do punho (horário) em um modelo de interface de maçaneta de porta (CAMPOS, 2010). 


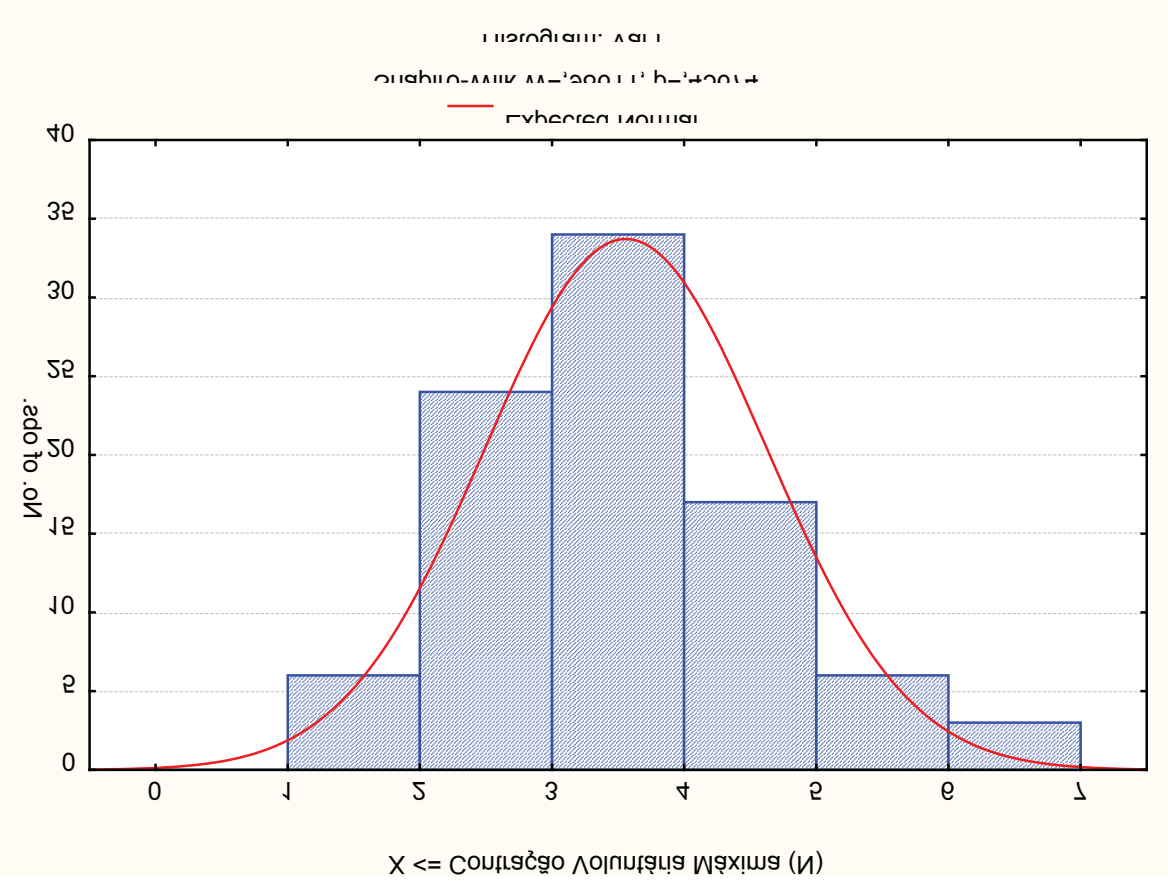

Figura 1. Polígono de frequências de dados de força manual em Newtons (N). Fonte: do autor

No Polígono de frequências é fácil localizar-se a Moda, ou seja, o valor amostral que foi observado com maior frequência. Outra vantagem da representação gráfica das distribuições é que ela permite constatar imediatamente os casos em que há duas ou mais modas, o que é um indicador de que a amostra é heterogênea, porque ela deve estar representando mais de uma população, neste caso ela apresentará o polígono de frequências similar ao que é apresentado na Figura 1. Se a amostra possui apenas uma moda é chamada de unimodal (BEIGUELMAN, 2002).

Os Histogramas também permitem que seja localizada a Mediana, isto é, a medida central do conjunto analisado. Cinquenta por cento (50\%) dos dados serão localizados a esquerda da Mediana e os outros 50\% à direita (BEIGUELMAN, 2002).

Segundo Tullis e Albert (2008), estas estatísticas, também conhecidas como Medidas de tendência central são a primeira coisa que deve ser observado ao executar estatística descritiva. Tendência central é simplesmente o meio, ou parte central de qualquer distribuição. As três medidas mais comuns de tendência central são estas, a média, mediana e moda.

Outra aplicação prática da distribuição de frequências é a verificação da normalidade das amostras, requisito essencial para a decisão por técnicas paramétricas ou não paramétricas, as quais serão apresentadas adiante. A 
distribuição normal é uma distribuição teórica de frequências muito importante e útil para a análise de variáveis continuas. Isso ocorre, pois são extremamente comuns nas características biológicas, expressas por variáveis contínuas, que mostram grande manipulação ajustamento à distribuição representada pela curva normal (BEIGUELMAN, 2002).

Os programas de análise estatística (SPSS ${ }^{\circledR}$, STATISTICA ${ }^{\circledR}$, BIOSTAT ${ }^{\circledR}$, dentre outros) bem como o Excel, efetuam a Distribuição de frequências de dados, o Histograma, o Polígono de frequências, bem como a Moda e a Mediana com bastante praticidade. Além disto, podem ser aplicados testes para verificação da normalidade, como por exemplo, o teste de Shapiro-Wilk. De um modo bastante simplificado, este teste retorna um resultado, através do qual é possível compreender se a amostra é homogênea ou não: caso do valor de "p" seja maior que 0,05 , significando que a amostra é homogênea. A importância de conhecer a normalidade das amostras implica resumidamente na definição do tipo de teste de inferência estatística para o caso da comparação das amostras, o qual será apresentado a seguir.

\subsection{Recomendações ao planejamento estatístico para pesquisas em design} Segundo Marconi e Lakatos (1996), as fases da pesquisa compreendem: 1. Escolha do tema; 2. levantamento de dados (pesquisa bibliográfica); 3. formulação do problema; 4. definição dos termos (conceitos a serem utilizados na pesquisa); 5. construção de hipóteses; 6. indicação de variáveis; 7. delimitação da pesquisa; 8. definição da amostragem; 9. seleção dos métodos e técnicas; 10. organização do instrumental da pesquisa; e 11. teste de instrumentos e procedimentos.

Uma das primeiras fases compreende a formulação do problema de pesquisa, o qual consiste em uma "dificuldade teórica ou prática, no conhecimento de alguma coisa de real importância, para qual se deve encontrar uma solução. Especificar um problema significa especificá-lo em detalhes precisos e exatos" (MARConi; LAKatos, 1996, p. 26). Ainda, segundo Marconi e Lakatos (1996, p. 26) "[...] O problema deve ser levantado, formulado, de preferência em forma interrogativa e delimitado com indicação das variáveis que intervém no estudo de possíveis relações entre si”.

\subsubsection{Variáveis de pesquisa}

As variáveis são os elementos constitutivos das hipóteses (LAKATOS; MARCONI, 1991). As hipóteses por sua vez, constituem-se em enunciados gerais de relações entre as variáveis (fatos, fenômenos), formulados como solução provisória para um determinado problema; apresentando caráter explicativo 
ou preditivo; compatível com o conhecimento científico (coerência externa) e revelando uma consistência lógica (coerência interna); sendo passível de verificação empírica em suas consequências (LAKATOS; MARCONI, 1991). Um importante conhecimento para o correto planejamento da análise estatística é o entendimento das variáveis da pesquisa. Existem diversas formas de classificar os tipos de variáveis, uma delas é enquadrando-as como dependentes ou independentes. A variável independente, segundo Lakatos e Marconi (1991, p. 172) é:

aquela que influencia, determina ou afeta a outra variável; é fator determinante, condição ou causa para certo resultado, efeito ou consequência; é o fator manipulado (geralmente) pelo investigador, na sua tentativa de segurar a relação do fator com o fenômeno observado ou a ser descoberto, para ver que influência exerce sobre um possível resultado.

Uma variável independente de um estudo é um aspecto manipulado, devem-se escolher as variáveis independentes com base na questão de pesquisa. Por exemplo, ao se preocupar com as diferenças de desempenho no uso de um produto entre homens e mulheres (Variáveis: Masculino x Feminino), ou entre novatos e especialistas (Variáveis: Novatos x Especialistas), ou entre dois projetos diferentes (Variáveis: Projeto A x Projeto B), todas estas são variáveis independentes que podem ser manipuladas para responder às perguntas de investigação específicas (TULLIS; ALBERT, 2008).

Já as variáveis dependentes consistem:

[...] naqueles valores (fenômenos ou fatores) a serem explicados ou descobertos, em virtude de serem influenciados, determinados ou afetados pela variável independente; é o fator que aparece desaparece ou varia à medida que o investigador introduz, tira ou modifica a variável independente; a propriedade ou o fator que é efeito, resultado, consequência ou resposta a algo que foi manipulado (variável independente) (LAKATOS; MARCONI, 1991, p. 172).

Variáveis dependentes (também chamadas de resultado ou variáveis de resposta) descrevem o que aconteceu como resultado do estudo. A variável dependente é algo que você mede como o resultado da (ou como dependente da) manipulação das variáveis independentes (TULLIS; ALBERT, 2008).

Segundo Tullis e Albert (2008), ao se planejar um estudo, deve-se ter ideia clara do que se pretende manipular (variáveis independentes) e do que 
se pretende medir (variáveis dependentes) (TULLIS; ALBERT, 2008). Além disso, conhecer estes tipos de variáveis é de grande importância no processo de análise estatística dos dados coletados.

Após definir as variáveis é importante definir quais tipos de dados serão coletados para posterior análise.

\subsubsection{Tipos de dados}

Os procedimentos estatísticos a serem utilizados dependem do tipo de variável e do tipo de dado coletado. Cada tipo de dado tem suas próprias forças e limitações, devem-se conhecer quais tipos de dados estão sendo tratados e que tipos de análises estatísticas podem ser realizados, pois cada tipo de dado requer um tratamento estatístico específico (TULLIS; ALBERT, 2008). Os autores descrevem alguns tipos de dados, os quais são recorrentes também na pesquisa em Design:

\section{a) Dados nominais}

Os dados nominais são grupos ou categorias desordenadas. Sem ordem entre as categorias, observa-se apenas que eles são diferentes, não que um seja melhor que o outro, mas apenas diferentes (TUlLis; ALBERT, 2008). Dados nominais podem ser: características de diferentes tipos de usuários, usuários em diferentes localizações geográficas, ou masculinos em oposição ao feminino. Estas são tipicamente variáveis independentes que permitem que se segmentem os dados por estes grupos diferentes (TULLIS; ALBERT, 2008). Os dados nominais também incluem variáveis dependentes, como por exemplo o número de participantes que escolheram o produto $\mathrm{A}$ em vez do produto $\mathrm{B}$ (TULLIS; ALBERT, 2008).

Entre os procedimentos estatísticos que podem ser usados com dados nominais encontram-se as simples estatísticas descritivas como contagens e frequências. Por exemplo, pode-se dizer que $45 \%$ dos participantes são do sexo feminino, ou $95 \%$ foram bem sucedidos em uma determinada tarefa, que 30\% escolheram o produto A e $70 \%$ o produto B, dentre outras (TULLIS; ALBERT, 2008).

\section{b) Dados ordinais}

Dados ordinais são ordenados em grupos ou categorias. Como o nome indica, os dados são organizados de modo que intervalos entre as medições não são significativos. Podem ser avaliados como dados classificados. Isto se deve ao fato de que distância entre as fileiras não é significativa, não é possível afirmar que uma categoria é duas vezes melhor que outra. Dados ordinais podem ser verificados nas avaliações de "melhor ou pior", "mais satisfeitos ou menos satisfeitos", ou, "mais grave ou menos grave". A classificação relativa (a ordem 
do ranking) é a única informação que importa. Em design, uma ocorrência comum de dados ordinais vem de medidas autorrelatadas, como os dados coletados por questionários. Por exemplo, um participante pode avaliar um produto como excelente, bom, regular ou ruim. Estas são posições relativas: a distância entre excelente e bom não é necessariamente a mesma distância entre o bem e o justo. A forma mais comum para analisar os dados ordinais é verificar as frequências. Por exemplo, você pode relatar que $40 \%$ dos participantes avaliaram o site como excelente, $30 \%$ como bom, $20 \%$ como justo, e $10 \%$ como ruim (TULLIS; ALBERT, 2008).

\section{c) Dados de intervalo}

Dados de intervalo ou intervalares são dados contínuos em que as diferenças entre as medições são significativas. Intervalos de dados permitem calcular uma ampla gama de estatísticas descritivas (incluindo médias, desvio padrão, etc.). Há também a possibilidade de aplicar estatística inferencial que pode ser usada para generalizar sobre uma população maior. Dados de intervalo fornecem mais possibilidades para a análise do que dados nominais ou ordinais. Um ponto importante neste sentido é o correto planejamento do instrumento de coleta de dados e a correta análise considerando o modo que esses dados foram coletados. Ao observar as escalas abaixo pode parecer que se tratam de escalas iguais, mas a diferença no modo de apresentação as torna diferentes (TULLIS; ALBERT, 2008). Segundo os autores, colocar rótulos explícitos sobre os itens da primeira escala a torna com dados ordinais. Deixar os rótulos fora, na segunda escala e apenas rotular o ponto final torna os dados de intervalo (Figura 2).

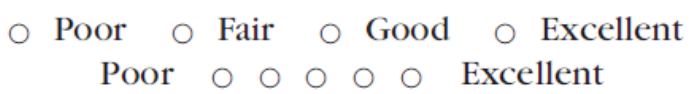

Figura 2. Escalas: ordinal e de intervalo. Fonte: Tullis e Albert (2008)

À primeira vista, pode-se dizer que as duas escalas são as mesmas, mas a diferença de apresentação as torna diferentes. Essa é a razão para que escalas mais subjetivas rotulem os fins, ou "âncoras", e não cada ponto de dados. 


\section{d) Dados de relação}

Finalmente, os dados de relação são os mesmos que os dados de intervalo, com a adição de um zero absoluto. Com dados de taxa, as diferenças entre as medidas são interpretadas como uma razão/relação. Exemplos de dados de relação são a idade, altura e peso. Em cada exemplo, zero indica ausência de idade, altura, ou peso. Não há muitas análises adicionais que você pode fazer com dados de relação em comparação com dados de intervalo (TULLIS; ALBERT, 2008).

\subsubsection{Procedimentos de análise}

Após conhecer as variáveis da pesquisa e definir os tipos de dados que serão coletados é possível partir para as próximas etapas de coleta e análise de dados. Neste ponto, escolher as estatísticas corretas é fundamental. Segundo Tulis e Albert (2008) decidir pelo teste errado e terminar com uma conclusão incorreta poderia invalidar os resultados e invalidar a avaliação inteira. Dependendo do tipo de dado coletado a análise pode compreender desde a apresentação dos dados através de estatísticas descritivas, até inferência estatística através de análises univariadas e até análises multivariadas. A seguir serão apresentadas algumas noções de análise para comparação de amostras através de estatística inferencial com análises univariadas.

\subsubsection{Comparação de amostras}

Um problema comum em inferência estatística é determinar, em termos de uma probabilidade, se diferenças observadas entre duas amostras significam que as populações correspondentes são realmente diferentes entre si. Ou seja, se ao comparar duas amostras as diferenças verificadas são estatisticamente significativas. Por exemplo, ao comparar as propostas de design de um produto percebidas por uma amostra de homens e uma amostra de mulheres, as diferenças observadas entre estes dois grupos são estatisticamente significativas? No desenvolvimento dos métodos de estatística moderna, as primeiras técnicas de inferência, as Paramétricas, foram baseadas na suposição de que os dados foram extraídos de uma população com Distribuição Normal. Um pouco mais recente assistiu-se o desenvolvimento de um grande número de técnicas de inferência, as quais não fazem suposições restringentes sobre a população da qual os dados são extraídos, essas técnicas são as Não paramétricas, ou livres de Distribuição Normal (SIEGEL; CASTELlan, 2006). 


\section{a) Técnicas paramétricas}

\section{Teste $\mathbf{t}$ de Student}

Segundo Arango (2005, p. 253) o teste t de Student "é o método mais usado para se avaliarem as diferenças entre as médias de dois grupos”, desde que "seja admitido que as populações que deram origem às amostras, tenham distribuição normal e variabilidades não significativamente diferentes”.

Quando as amostras a serem comparadas forem normais e para variáveis independentes aplica-se o Teste t. Algumas das modalidades desse teste são:

- Teste $t$, independente, por grupos: utilizado para comparar respostas de homens e mulheres em uma pesquisa, por exemplo. Os dados são organizados na mesma coluna e identificados por grupos;

- Teste $t$, amostras dependentes: compare as respostas da mesma pessoa quanto a questões de uma pesquisa antes e depois de um evento;

De modo simplificado, a interpretação destes resultados deverá ser realizada da seguinte forma: quando $p>0,05$, não há diferenças estatisticamente significativas na comparação entre as amostras. Quando o $\mathrm{p} \leq 0,05$ há diferenças estatisticamente significativas na comparação das amostras.

\section{ANOVA}

É utilizada para comparar mais de duas amostras, quando estas apresentam normalidade de variâncias. O resultado da ANOVA apresenta se há diferenças significativas nas comparações realizadas, mas não identifica onde essas diferenças ocorrem. Para isso é necessário aplicar um teste Post-Hoc.

\section{b) Técnicas não paramétricas}

Quando a normalidade é descartada em todas as amostras a serem comparadas ou em uma delas, mesmo que a (as) outra (as) apresentem normalidade, recorre-se aos testes não paramétricos, os mais aplicados são:

\section{Wilcoxon}

É utilizado para comparar um par de amostras dependentes (variáveis). Ele é projetado para testar uma hipótese sobre a localização (mediana) de uma distribuição da população. Muitas vezes envolve o uso de pares correspondentes, por exemplo, "antes" e "depois" (amostras emparelhadas). Pode ser utilizada na comparação de duas situações verificadas para o mesmo sujeito, antes x depois, ou produto A x B avaliados pelo mesmo sujeito. 


\section{Mann-Whitney}

É utilizado para comparar duas amostras independentes (grupos), como por exemplo, diferenças entre gêneros. Conforme Arango (2005) é apropriado "quando se tem um par de amostras independentes e se deseja testar se as populações que deram origem a essas amostras podem ser consideradas semelhantes ou não" (p. 277), ou seja, se entre elas existem, ou não, diferenças estatisticamente significativas $(\mathrm{p} \leq 0,05)$.

Quando há normalidade nas amostras, mas apenas uma delas a serem comparadas for homogênea para amostras independentes aplica-se o Mannwhitney para amostras emparelhadas aplica-se o Wilcoxon.

\section{Friedman}

É utilizado para comparar múltiplas amostras emparelhadas (três ou mais). Pode ser utilizada na comparação de mais de duas situações verificadas para o mesmo sujeito, antes x durante x depois, ou produto A x B x C. Assim como na ANOVA o teste de Friedman apresenta se há diferenças significativas nas comparações, entretanto não apresenta onde estas diferenças ocorrem. Para isso é necessário aplicar o teste Post-Hoc de Dunn.

\section{Kruskal-Wallis}

É utilizado para comparar múltiplas amostras (três ou mais) independentes (grupos). Consiste em um teste não paramétrico alternativo à ANOva (one-way). É utilizado para comparar três ou mais amostras. Pode ser utilizado para comparar faixas etárias, por exemplo, distribuídas em pelo menos três grupos.

Um resumo de algumas noções de possíveis procedimentos estatísticos adequados a cada tipo amostra, pode ser verificado no diagrama apresentado na Figura 3. 


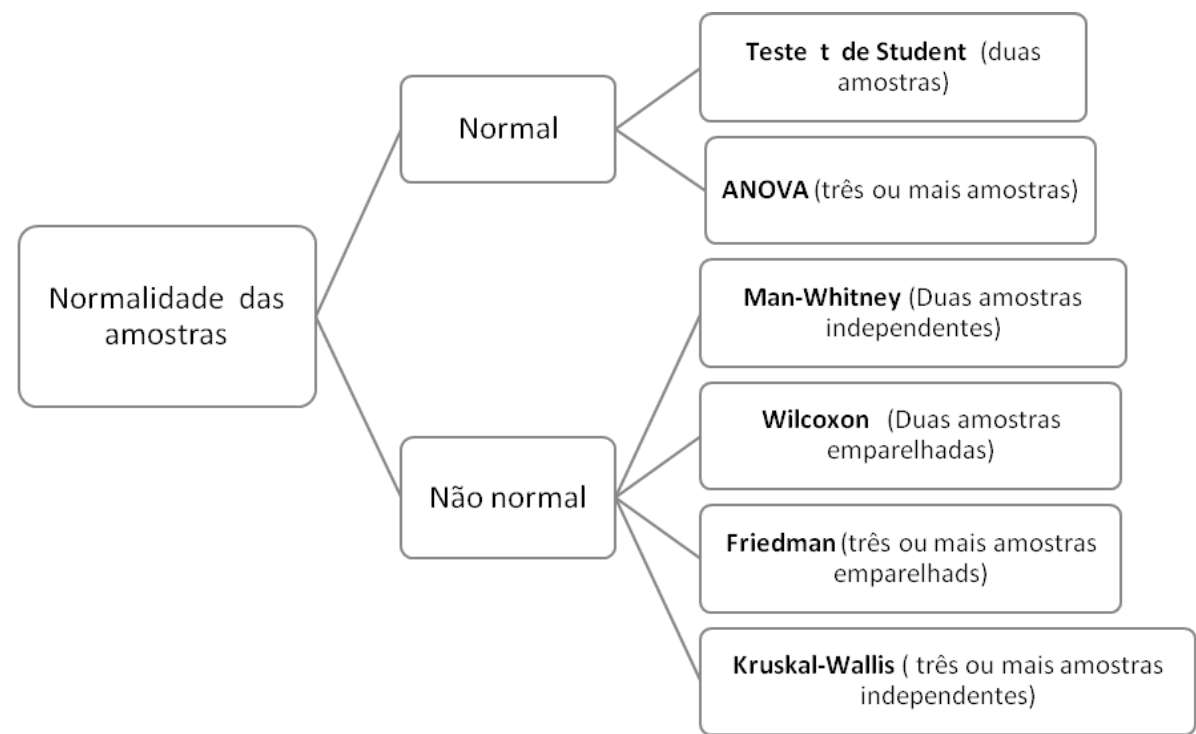

Figura 3. Diagrama resumo de alguns procedimentos para análise estatística de comparação de amostras.

\section{Considerações Finais}

Em suma, observa-se que o conhecimento da estatística pode ser crucial em Pesquisas, assim com na área do Design. Há procedimentos que podem ser encarados como simples e que são importantes para a validação de hipóteses, como no caso de pesquisas descritivas, que relatam dados encontrados por frequência de respostas. Por outro lado, também, é possível a aplicação de procedimentos mais complexos, que utilizam a correlação entre variáveis inerentes a hipóteses, como em pesquisas experimentais. Ambos os procedimentos, dependem de uma logística estratégica, em termos de processos estatísticos, a qual deve ser elaborada pelo pesquisador em design tendo como referência a formulação do problema encontrado (demanda de Design).

Outra questão influente, quanto à logística supracitada, é que a Pesquisa em Design pode ser considerada como um contexto multidisciplinar e, assim, pode ser encarada como uma referência às modificações que vem ocorrendo na área de Design nas últimas décadas. É fato que, nos primeiros estudos, durante a década de 1960, a metodologia projetual foi o principal foco, sendo que, a ênfase que este tema teve por muitos anos, levou a confundir Pesquisa em Design com a pesquisa para a prática do design (VAN DER LINDEN, 2010). Com base nesta afirmação, pode-se inferir que o fator Multidisciplinaridade, comum na área de Design, pode ser o norteador da dificuldade de realização e aplicação da pesquisa na referida área, incluindo a aplicação de processos estatísticos. Por outro lado, considera-se que o aspecto multifacetado do Design 
pode determinar estratégias estatísticas específicas, ou seja, cada faceta (produto, gráfico, informação, meios eletrônicos e digitais, ambientes construídos, têxtil e de vestuário, ergonomia e usabilidade, sustentabilidade, aspectos artísticos, filosóficos, sociais e comunicacionais) pode possuir uma maneira peculiar de uso e aplicação da estatística.

Vale ressaltar, também, que o conhecimento em Estatística é, portanto, indispensável na produção de conhecimento científico, sistemático e verificável. Mesmo que o uso e aplicabilidade da estatística tenham vieses diferentes, em pesquisas científicas e em pesquisas advindas do mercado. Tal fato traz o questionamento a respeito da contratação de um profissional da estatística em pesquisas da área de Design (científicas ou técnicas, de mercado) e o nível de confiabilidade do planejamento quanto ao processo estatístico e quanto à validação dos resultados encontrados, além de sua aplicabilidade real. Vale a recomendação de que o Designer deve ter um conhecimento base (mínimo) sobre os processos estatísticos para poder trabalhar em conjunto com o Profissional da estatística, desta forma, construindo uma possibilidade maior de validação, em termos de estatística, e confiabilidade de resultados. O mesmo pode ser pensado a respeito das bases do conhecimento estatístico (estatística e probabilidade), no que diz respeito ao oferecimento das disciplinas relativas à estatística na graduação e na pós-graduação de design: qual a real estratégia didático-pedagógica para que o aluno dos cursos de Design possuam conhecimento base sobre processos estatísticos aplicados ao Design?, Tal reflexão é pertinente para que se possa, de maneira contundente, se pensar na estatística como um conhecimento específico que pode implementar a pesquisa em Design.

\section{Referências Bibliográficas}

Beiguelman, B. Curso Prático de Bioestatística. Ribeirão Preto: Fundação de Pesquisas Científicas de Ribeirão Preto, 2002.

BONSIEPE, G. Design: com prática de projeto. São Paulo: Blucher, 2014p. 2012.

BONFIM, G. A. Idéias e formas na história do design: uma investigação estética. João Pessoa: Editora Universitária (UFPв), 185p., 1998.

CHANG, W; WU, T. Exploring Types and Characteristics of Product Forms. International Journal of Design, 1(1), p. 3-14, 2007. 
BLIJLEVENS, J., MUGGE, R., Y, P., SCHOORMANS, J. P. L. The influence of product exposure on trendiness and aesthetic appraisal. International Journal of Design, 7(1), p. 55-67, 2013.

FORSLUND, K., KARLSSON, M., SÖDERBERG, R. Impacts of geometrical manufacturing quality on the visual product experience. International Journal of Design, 7(1), p. 69-84, 2013.

GROENESTEIJN, L; EIKHOUT, S. M.; VINK, P. One set of pliers for more tasks in installation work: the effects on (dis)comfort and productivity. Applied Ergonomics, (35), p. 485-492, 2004.

KIM, S. Y., CHO, K. Usability and design guidelines of smart canes for users with visual impairments. International Journal of Design, 7(1), p. 99-110, 2013.

LAKATOS, E. M.; MARCONI, M.A. Metodologia Científica. 2ed. São Paulo: Atlas, 1991.

LÖBACH, B. Design Industrial - Bases para a configuração dos produtos industriais; tradução Freddy Van Camp. São Paulo: Editora Blucher, 206p. 2001.

MARCONI, M. DE A.; LAKATOS, E M. Técnicas de pesquisa: planejamento e execução de pesquisas, amostragens e técnicas de pesquisa, elaboração, análise e interpretação de dados. $3^{\text {a }}$ Ed. São Paulo: Atlas, 231p. 1996.

MORAES, A. DE; MONT'ALVÃO, C. Ergonomia: conceitos e aplicações. $4^{\mathrm{a}}$ Ed. Rio de Janeiro: 2AB, 224p. 2010.

NIEMEYER, L. Pesquisa em design no Brasil: possibilidades e desafios. In: II Seminário de Metodologia em Design. Recife: Programa de Pós-Graduação em Design (UFPE). 2008.

PASCHOARELLI, L. C.; SILVA, J. C. P.; CAMPOS, L. F. A.; MENIN, M.; SILVA, J. C. R. P.; LANUTTI,J. N. L.; MUNIZ, F. J. A contribuição do design e das artes gráficas na pesquisa e desenvolvimento em design no Brasil. Anais do CIGAG 2010. Lisboa, 2010.

TULLIS, T.; ALBERT, B. Measuring the user experience: collecting, analyzing and presenting usability metrics. Burlington: Morgan Kaufmann, 2008. 
VAN DER LINDEN, J. Uma taxonomia para a Pesquisa em Design. In: $\mathbf{9}^{\mathbf{0}}$ Congresso Brasileiro de Pesquisa e Desenvolvimento em Design ( $9^{\circ} \mathrm{P} \& \mathrm{D}$ Design). São Paulo: UAM. 2010.

VERGARA, M.; MONDRAGÓN, S.; SANCHO-BRU, J. L.; COMPANY, P.; AGOST, M. Perception of products by progressive multisensory integration. A study on hammers. Applied Ergonomics, (42), p. 652 - 664, 2011.

\section{Agradecimentos}

Os autores agradecem a participação dos alunos de iniciação científica Samuel Renato e Paula Maia pela contribuição no levantamento de dados quanto às referências bibliográficas utilizadas no presente artigo. 
Recebido em: 18/03/2013

Aceito em: 01/05/2013

\section{Como citar}

DINIZ, R. L.; CAMPOS, L. F. A.; NICÁCIO, P. R. S.; SILVA FILHO, O. L. A importância da estatística para a pesquisa em Design: algumas recomendações. Arcos Design. Rio de Janeiro: PPD ESDI - UERJ. Volume 7 Número 1 Junho 2013. pp. 157-177. Disponível em: [http://www.e-publicacoes.uerj.br/index.php/arcosdesign]

DOI

10.12957/arcosdesign.2013.10003

\section{(1) (1)(2)}

A Revista Arcos Design está licenciada sob uma licença Creative Commons Atribuição - Não Comercial - Compartilha Igual 3.0 Não Adaptada. 